\title{
A CONDITION ON THE VALUE FUNCTION BOTH NECESSARY AND SUFFICIENT FOR FULL REGULARITY OF MINIMIZERS OF ONE-DIMENSIONAL VARIATIONAL PROBLEMS
}

\author{
M. A. SYCHEV AND V. J. MIZEL
}

ABstract. We study two-point Lagrange problems for integrands $L=$ $L(t, u, v)$ :

$(\mathrm{P})$

$$
\begin{aligned}
F[u]=\int_{a}^{b} L(t, u(t), \dot{u}(t)) d t & \rightarrow \inf , \\
u & \in \mathcal{A}=\left\{v \in W^{1,1}\left([a, b] ; \mathbb{R}^{n}\right) \mid v(a)=A, v(b)=B\right\} .
\end{aligned}
$$

Under very weak regularity hypotheses $[L$ is Hölder continuous and locally elliptic on each compact subset of $\left.\mathbb{R} \times \mathbb{R}^{n} \times \mathbb{R}^{n}\right]$ we obtain, when $L$ is of superlinear growth in $v$, a characterization of problems in which the minimizers of $(\mathrm{P})$ are $C^{1}$-regular for all boundary data. This characterization involves the behavior of the value function $S: \mathbb{R} \times \mathbb{R}^{n} \times \mathbb{R} \times \mathbb{R}^{n} \rightarrow \mathbb{R}$ defined by $S(a, A, b, B)=\inf _{\mathcal{A}} F$. Namely, all minimizers for (P) are $C^{1}$-regular in neighborhoods of $a$ and $b$ if and only if $S$ is Lipschitz continuous at $(a, A, b, B)$. Consequently problems (P) possessing no singular minimizers are characterized in cases where not even a weak form of the Euler-Lagrange equations is available for guidance. Full regularity results for problems where $L$ is nearly autonomous, nearly independent of $u$, or jointly convex in $(u, v)$ are presented.

\section{INTRODUCTION}

We consider here the two-point Lagrange problem

$$
\begin{aligned}
F[u]=\int_{a}^{b} L(t, u(t), \dot{u}(t)) d t & \rightarrow \inf , \\
u & \in \mathcal{A}=\left\{v \in W^{1,1}\left([a, b] ; \mathbb{R}^{n}\right) \mid v(a)=A, v(b)=B\right\} .
\end{aligned}
$$

We define the value function $S: \mathbb{R} \times \mathbb{R}^{n} \times \mathbb{R} \times \mathbb{R}^{n} \rightarrow \mathbb{R}$ as follows:

$$
S(a, A, b, B)=\inf \{F[u] \mid u \in \mathcal{A}\} .
$$

Received by the editors August 17, 1995 and, in revised form, December 28, 1995.

1991 Mathematics Subject Classification. Primary 49N60, 49L99, 49J45.

Key words and phrases. Tonelli partial regularity result, conditionally equicontinuous family, value function.

Research of the first author partially supported by the NSF under Grant DMS9320104 and by the grant "Lavrentiev's effect and applications" of the Siberian Division of the Russian Academy of Science.

Research of the second author partially supported by the NSF under Grant DMS9201221. 
It is well known that problem (1) has a minimizer ["solution"] if $L=L(t, u, v): \mathbb{R} \times$ $\mathbb{R}^{n} \times \mathbb{R}^{n} \rightarrow \mathbb{R}$ is convex in $v$ and satisfies the following growth condition: $L(t, u, v) \geq$ $\mathcal{O}(v)$, where $\mathcal{O}(v) /|v| \rightarrow \infty$ as $|v| \rightarrow \infty$. This growth condition ensures relative weak compactness of subsets of $\mathcal{A}$ satisfying $F[u] \leq M<\infty$, for fixed $M>0$. Thus for $M$ sufficiently large there exists in each such subset a minimizing sequence $\left\{u_{n}\right\}$ converging weakly to some function $u_{0} \in \mathcal{A}$. The convexity in $v$ of $L$ ensures lower semicontinuity of $F$ with respect to sequential weak convergence, so that $\liminf _{n \rightarrow \infty} F\left[u_{n}\right] \geq F\left[u_{0}\right]$, whence $u_{0}$ is a minimizer for (1). Moreover, if $L$ is $C^{1}$ and is strictly convex in $v$, then any such minimizer $u_{0}$ is itself $C^{1}$ on some open subset $\Omega$ having full measure in $[a, b]$, satisfies the Euler-Lagrange equation $d / d t L_{v}\left(t, u_{0}(t), \dot{u}_{0}(t)\right)=L_{u}\left(t, u_{0}(t), \dot{u}_{0}(t)\right)$ on $\Omega$ and $\left|\dot{u}_{0}(t)\right| \rightarrow \infty$ as $t \rightarrow t_{0} \in$ $[a, b] \backslash \Omega$ (Tonelli's partial regularity theorem [T, p. 345], [BM], [CV1], [CV2], [S1]).

These properties, when allied with an absence of bounded solutions of the EulerLagrange equation with unbounded derivative, provide full regularity for all minimizers. This was the main idea of the approach in [CV1]. There full regularity of solutions was proved for problems with autonomous $L$, but the approach used there did not have the character of a necessary and sufficient condition. There are nonautonomous integrands $L$ for which the minimizers of problem (1) for all $(a, A, b, B)$ are $C^{1}$-regular, while there exist bounded solutions with unbounded derivative of the corresponding Euler-Lagrange equations $[\mathrm{BM}]$, [S2]. Such solutions of the Euler-Lagrange equations don't have "variational character". This fact is not surprising, since it is well known that even classical solutions of these equations can fail to be local minimizers of $F$. In order to clarify when a classical solution of the Euler-Lagrange equations is a local minimizer, it was necessary to develop the field theory of the calculus of variations [Bo], [Ca], [Ce]. Clearly, a field theory for problems with nonclassical solutions (solutions satisfying only the requirements of Tonelli's partial regularity theorem) will be considerably more complicated.

Thus in the present article we bypass this issue by characterizing regularity for solutions of (1) in terms of qualitative properties of the value function $S$ defined in (2). It turns out that Lipschitz continuity of $S$ is such a property. Moreover we will present a method which shows how this result can be used to prove full regularity in the presence of such mild restrictions on the integrand that even the very weak version of the Euler-Lagrange equation utilized in [CV1] is unavailable.

We begin by proving a partial regularity theorem, as well as theorems concerning existence and regularity in the small, under conditions on $L$ which are weaker than usual. We adopt the following assumptions concerning the integrand:

$L: \mathbb{R} \times \mathbb{R}^{n} \times \mathbb{R}^{n} \rightarrow \mathbb{R}$ is Hölder continuous on each compact subset $G \subset \mathbb{R} \times \mathbb{R}^{n} \times \mathbb{R}^{n}$, and $L=L(t, u, v)$ is convex in $v$. In addition, for each such subset $G$ there exists a positive number $\mu=\mu(G)$ such that $L\left(t, u, v_{2}\right)-L\left(t, u, v_{1}\right)-\left\langle l, v_{2}-v_{1}\right\rangle \geq \mu\left|v_{2}-v_{1}\right|^{2}$ holds for each $\left(t, u, v_{1}\right),\left(t, u, v_{2}\right) \in G$ and each $\left.l \in \partial L(t, u, \cdot)\right|_{v=v_{1}}$.

We will need to extend certain definitions from [S3] as follows.

Definition. Consider a family of functions $\mathcal{X}=\left\{\xi:[a, b] \rightarrow \mathbb{R}^{n}\right\}$ each of which is continuous on some open subset $\Omega=\Omega(\xi) \subset[a, b]$ of full measure and satisfies for each $t_{0} \in[a, b] \backslash \Omega(*) \lim _{t \in \Omega, t \rightarrow t_{0}}|\xi(t)|=\infty$.

Such a family $\mathcal{X}$ is said to be a conditionally equicontinuous family $(\mathrm{CEF})$ if for each $M>0$ and $\varepsilon>0$ there exists $\delta(M, \varepsilon)>0$ such that if $\xi \in \mathcal{X}$ and $t_{0} \in \Omega(\xi)$ with $\left|\xi\left(t_{0}\right)\right|<M$ then $\left|t-t_{0}\right| \leq \delta(M, \varepsilon)$ implies $t \in \Omega(\xi)$ and $\left|\xi(t)-\xi\left(t_{0}\right)\right| \leq \varepsilon$. 
Any such function $\delta$ is referred to as a conditional equicontinuity modulus function $(\mathrm{CEMF})$ for the family $\mathcal{X}$.

Sometimes we will also consider families of functions $\{\xi\}$ defined on different closed intervals.

Theorem 1. Suppose that $L$ satisfies (H1), $K$ is a compact subset of $\mathbb{R} \times \mathbb{R}^{n}$ and $M>0$. Then there exists $\varepsilon>0$ such that, for each $(a, A) \in K$, if $(b, B)$ satisfies $|B-A| /|b-a| \leq M$ then for some $\gamma>0$ problem (1) has only $C^{1, \gamma}$-regular minimizers in the class of functions in $\mathcal{A}$ satisfying $\left|u_{i}(t)-A_{i}\right| \leq \varepsilon, t \in[a, b]$, $1 \leq i \leq n$, provided that $|b-a| \leq \eta(M, \varepsilon)$ is sufficiently small. Moreover, the derivatives of all such minimizers form a bounded set in $C^{0, \gamma}$.

Theorem 2. Suppose that L satisfies (H1) and $K$ is a compact subset of $\mathbb{R} \times \mathbb{R}^{n}$. Let $U$ be the collection of all solutions of problems of the form (1) whose graphs lie in $K$. Then $V=\{\dot{u} \mid u \in U\}$ is a CEF.

Our main result is the following:

Theorem 3. Suppose that $L=L(t, u, v)$ satisfies (H1) and has superlinear growth in $v[L(t, u, v) \geq \vartheta(v) \geq 0$, where $\vartheta(v) /|v| \rightarrow \infty$ as $|v| \rightarrow \infty]$. Then $S: \mathbb{R} \times$ $\mathbb{R}^{n} \times \mathbb{R} \times \mathbb{R}^{n} \rightarrow \mathbb{R}$ is Lipschitz continuous at a point $\left(a_{0}, A_{0}, b_{0}, B_{0}\right)$ if and only if all solutions of (1) with boundary condition $(a, A, b, B)=\left(a_{0}, A_{0}, b_{0}, B_{0}\right)$ have bounded derivatives at $a$ and $b$.

Corollary. If $L$ satisfies the conditions of Theorem 3 then problem (1) has only $C^{1}$-regular minimizers for all boundary conditions if and only if the value function $S$ is Lipschitz continuous at every point.

We prove Theorems 1 and 2 in $\S 3$ and Theorem 3 in $\S 4$. In $\S 2$ we prove some properties of CEF's which are needed for the proofs of Theorems 1-3. In $\S 5$ we demonstrate how arguments used in the proof of Theorem 3 simplify in proving regularity for problems with weak $t$ - or $u$-dependence as well as for integrands which are jointly convex in $(u, v)$ (cf. Theorem 4 in $\S 5$ ). In these cases it suffices to prove the Lipschitz property of $S$ in directions involving variation of a single argument rather than proving its full Lipschitz continuity. We note that the Lipschitz condition in Theorem 3 could be replaced in general by a Lipschitz property with respect to special directions (cf. the Remark following the proof) at the cost of significantly complicating the statement of the theorem.

It is worth mentioning that there is some hope of obtaining necessary and sufficient conditions for the nonoccurrence of the Lavrentiev gap phenomenon in terms of the regularity of $S$. Recall that this gap phenomenon [L], [Ma], [BM] refers to problems in which a global regularity constraint results in an increase in the infimum of a variational problem. Lavrentiev $[\mathrm{L}]$ provided the first example of problem (1) in which the integrand $L$ and class $\mathcal{A}$ were such that

$$
m=\inf _{\mathcal{A} \cap C^{1}} F>S(a, A, b, B)=\inf _{\mathcal{A}} F .
$$

Since that time other cases have been studied [BM], [Da] in which

$$
m_{p}=\inf _{\mathcal{A} \cap W^{1, p}} F>S(a, A, b, B), \quad \text { for some } p>1 .
$$

One possibility is that the key issue is continuity of $S$ (cf. [FS, Ch1]): it appears that continuity of $S$ everywhere follows from the absence of a Lavrentiev gap for all problems (1) with the given integrand. 
A second important problem is to resolve the question alluded to earlier: when does a "solution" of the Euler-Lagrange equation which satisfies Tonelli's partial regularity conditions yield a minimizer for (1)? It seems clear that resolving this matter will necessitate the development of a field theory for problems with singular minimizers. Here, in contrast to the classical case in which $C^{1}$-norms of functions are bounded, it is not enough to know the values of the integrands and their partial derivatives at the graph of the function under consideration in determining whether the function is a minimizer. In an example from $[\mathrm{S} 1, \S 2] L=\mu v^{2}+\left(u^{5}-t^{3}\right)^{4} v^{32}$ and the function $u_{0}(t)=k t^{3 / 5}, k=k(\mu)$, is a solution for any problem with boundary conditions taken from its graph, but [for boundary conditions with $a \leq 0, b \geq 0$ ] is not a minimizer for the integrand of [S2, Theorem 1]

$$
L=\mu v^{2}+\left(t^{3}-u^{5}\right)_{+}^{4} p\left(\left(u^{5} / t^{3}-k_{0}^{5}\right) /\left(\gamma_{0}-k_{0}\right)\right) v^{32},
$$

with appropriately chosen $p \in C^{\infty}[p(t)=0, t \leq 0, p(t)=1, t \geq 1], k_{0}<k, \gamma_{0}=$ $\left(k+k_{0}\right) / 2$, despite the fact that both integrands have the same values and partial derivatives at the graph of $u_{0}$.

\section{Some PROperties of CEF's}

Lemma 2.1. Let $\mathcal{X}=\{\xi\}$ be a CEF with CEMF $\delta$. Then

(1) Each $L_{1}$ bounded sequence $\left\{\xi_{n}\right\} \subset \mathcal{X}$ has a subsequence which converges uniformly to a function $\xi_{0}$ over every compact subset of some open subset $\Omega$ of full measure in the interval of definition; moreover $\mathcal{X} \cup\left\{\xi_{0}\right\}$ is a CEF with the same CEMF $\delta$.

(2) Subsets of $\mathcal{X}$ which are relatively weakly compact in $L_{1}$ are relatively strongly compact in $L_{1}$; moreover $\mathcal{X}$ together with all its $L_{1}$ sequential weak limits is also a $C E F$ with the same CEMF $\delta$.

The proof is the same as that for Lemma 2.1 of [S3].

Lemma 2.2. Let $\mathcal{X}$ be a CEF and suppose that $\left\{\xi_{m}\right\}_{0 \leq m} \subset \mathcal{X}$ is such that $\left\|\xi_{m}\right\|_{L_{1}(a, b)} \leq C<\infty$, and the functions $f_{m}:[a, b] \rightarrow \mathbb{R}^{n}, m \geq 1$, defined by $f_{m}(t)=\int_{a}^{t}\left[\xi_{m}(s)-\xi_{0}(s)\right] d s$ converge pointwise to 0 as $m \rightarrow \infty$. Then

$$
\left\|\xi_{m}-\xi_{0}\right\|_{C(K)} \rightarrow 0
$$

for each compact subset $K$ of the open set $\Omega=\Omega\left(\xi_{0}\right)$. Moreover, if $M>0, \varepsilon>0$ and $E_{M}=\left\{t|| \xi_{0}(t) \mid \geq M+\varepsilon\right\}$, then $\lim _{m \rightarrow \infty}\left[\inf _{t \in E_{M}}\left|\xi_{m}(t)\right|\right]>M$.

Proof. In (1) and (2) we first prove pointwise convergence of $\xi_{m}$ to $\xi_{0}$.

(1) Suppose $t_{m} \rightarrow t_{0}$ are such that $\left|\xi_{m}\left(t_{m}\right)\right|,\left|\xi_{0}\left(t_{0}\right)\right|<M$. Then by the definition of CEF $\xi_{m}\left(t_{0}\right)-\xi_{m}\left(t_{m}\right) \rightarrow 0$. We prove that $\xi_{m}\left(t_{m}\right) \rightarrow \xi_{0}\left(t_{0}\right)$ by contradiction. If convergence fails there exists a subsequence $\left\{\xi_{k^{\prime}}\right\}$ such that $\xi_{k^{\prime}}\left(t_{0}\right) \rightarrow B \neq$ $\xi_{0}\left(t_{0}\right)$. Hence for some $\varepsilon>0$ and $i \in\{1, \ldots, n\},\left|\xi_{k^{\prime}}^{i}\left(t_{0}\right)-\xi_{0}^{i}\left(t_{0}\right)\right|>2 \varepsilon$ for all sufficiently large $k^{\prime}$. It now follows from the definition of CEF that for some $\delta>0$ $\left|\xi_{k^{\prime}}^{i}(t)-\xi_{0}^{i}(t)\right|>\varepsilon$ for all $t \in I=\left[t_{0}-\delta, t_{0}+\delta\right]$, and $\xi_{k^{\prime}}, \xi_{0}$ are equibounded and equicontinuous functions on $I$ ( $k^{\prime}$ sufficiently large). Thus

$$
\left\|f_{k^{\prime}}\left(t_{0}+\delta\right)-f_{k^{\prime}}\left(t_{0}-\delta\right)\right\|=\left\|\int_{t_{0}-\delta}^{t_{0}+\delta}\left[\xi_{k^{\prime}}(t)-\xi_{0}(t)\right] d t\right\| \geq \varepsilon \delta,
$$

which contradicts the hypothesis that $f_{k^{\prime}} \rightarrow 0$ pointwise. 
(2) Suppose $\left|\xi_{0}\left(t_{0}\right)\right|<M$ and put $J=\left[t_{0}-\delta, t_{0}+\delta\right]$, where $2 \delta<\delta(M+1,1)$. In view of (1) it suffices to prove that $\left|\xi_{m}\left(t_{m}\right)\right|$ is a bounded sequence whenever $t_{m} \rightarrow t_{0}$. Now by Chebychev's inequality for each $\xi_{m}$ there is a point $\tau_{m} \in J$ such that $\left|\xi_{m}\left(\tau_{m}\right)\right|<N$ with a prescribed $N>C / \delta$. By passing to a subsequence one can suppose that $\tau_{k^{\prime}} \rightarrow \tau_{0} \in J$. Hence by (1) it follows that $\xi_{k^{\prime}}\left(\tau_{k^{\prime}}\right) \rightarrow \xi_{0}\left(\tau_{0}\right)$, so that $\left|\xi_{k^{\prime}}\left(\tau_{k^{\prime}}\right)\right|<M+1$ for all sufficiently large $k^{\prime}$, and consequently $\left\|\xi_{k^{\prime}}\right\|_{C(J)} \leq M+2$ for all sufficiently large $k^{\prime}$. Since every subsequence of the original sequence possesses a subsequence of the type above, it follows that the full sequence $\left\{\xi_{k}\right\}$ satisfies $\left\|\xi_{k}\right\|_{C(J)} \leq M+2$ for all sufficiently large $k$. Hence the sequence $\left|\xi_{m}\left(t_{m}\right)\right|$ is bounded as claimed.

(3) If $K$ is a compact subset of $\Omega$ then the restriction of $\xi_{0}$ to $K$ is (uniformly) continuous, so that for some $M>0,\left|\xi_{0}(t)\right| \leq M$ for all $t \in K$, and by (2) $\xi_{k}(t) \rightarrow \xi_{0}(t)$ for each $t \in K$. In view of the definition of CEF one can deduce that for $k_{0}$ sufficiently large one has uniform boundedness of $\left\{\xi_{k}\right\}_{k \geq k_{0}}$ on $K$. Namely, there is a finite subset $T=\left\{\tau_{i}\right\}$ of $K$ such that each point of $K$ is within $\delta(M+1,1)$ of $T$. Since for $k_{0}$ sufficiently large one has $\left|\xi_{k}\left(\tau_{i}\right)\right|<M+1, \forall \tau_{i} \in T$ for each $k \geq k_{0}$, it follows that $\left\|\xi_{k}\right\|_{C(K)} \leq M+2$, for all $k \geq k_{0}$. Hence since $\mathcal{X}$ is a CEF these functions are equicontinuous on $K$, and thus their pointwise convergence to $\xi_{0}$ implies uniform convergence.

(4) Let $M>0, \varepsilon>0$, and $E_{M}=\left\{t|| \xi_{0}(t) \mid \geq M+\varepsilon\right\}$. If $\liminf _{k \rightarrow \infty, t \in E_{M}}\left|\xi_{k}(t)\right|$ $<M+\varepsilon / 2$, then one can extract a subsequence $t_{k^{\prime}} \rightarrow t_{0}$ such that $\xi_{k^{\prime}}\left(t_{k^{\prime}}\right) \rightarrow A$ with $|A| \leq M+\varepsilon / 2$ and $M+\varepsilon \leq\left|\xi_{0}\left(t_{0}\right)\right|<\infty$, which contradicts (1). This completes the proof.

\section{Proofs of Theorems 1 And 2}

Proof of Theorem 1. In (1) below we examine problem 1 where the admissible functions are constrained to lie in a bounded subset of $W^{1, \infty}$. In (2) we eliminate this constraint, utilizing stronger hypotheses on $L$. Then in (3) we reduce the hypotheses on $L$ to (H1).

(1) By (H1) one can choose $\varepsilon>0$ in such a manner that for some $\alpha>0, \beta \in \mathbb{R}$ one has for each $(a, A) \in K$ and each $\left.l \in \partial L(a, A, \cdot)\right|_{v=0}$

$$
L(t, u, v)-L(t, u, 0)-\langle l, v\rangle \geq \alpha|v|+\beta \quad \forall v \in \mathbb{R}^{n}
$$

for all $(t, u)$ satisfying

$$
(t, u) \in \mathbf{G}(a, A ; \varepsilon):=\left\{(s, r)|| s-a|\leq \varepsilon,| r_{i}-A_{i} \mid \leq \varepsilon, i=1, \ldots, n\right\} .
$$

Let us now restrict the corresponding functional $F$ to the class $\mathbf{G}$ of $W^{1, \infty}$ functions which satisfy for some $(a, A),(b, B), u(a)=A, u(b)=B$, with $(a, A) \in K$, $|B-A| /|b-a| \leq M,|b-a| \leq \eta$, and whose graphs lie in $\mathbf{G}(a, A ; \varepsilon)$. Given $N>0$ examine the minimization problem for $F$ on $\mathbf{G}_{N}:=\{u \in \mathbf{G}|| \dot{u}(t) \mid \leq N$ a.e. $\}$. Denoting this problem by $\mathrm{P}_{N}$, let us select $N_{1}$ such that for some $\beta^{*} \in \mathbb{R}$

$$
L(t, u, v)-\langle l, v\rangle \geq \alpha|v|+\beta^{*} \geq L(t, u, w)-\langle l, w\rangle
$$

is satisfied for all $|v| \geq N_{1},|w| \leq M,(t, u) \in \mathbf{G}(a, A ; \varepsilon), l \in \partial L(a, A, 0)$. In addition, we may choose $N_{1}$ sufficiently large that (3.2) holds uniformly with respect to $(a, A) \in K$. Furthermore, we can work with the integrands $L(t, u, v)-\langle l, v\rangle$ rather than $L$ because the integral of the linear form has the same value for all admissible functions satisfying fixed boundary conditions. 
For fixed $N$, problem $\mathrm{P}_{N}$ has only $C^{1}$-regular solutions ([S1, Theorem 1.2, Corollary 1]). The conditions on $L$ in Theorem 1.2 of [S1] were more restrictive than used here, but as mentioned at the end of $\S 1$ of [S1], the original proof applies to the present context. Moreover, Theorem 1.2 allows us to conclude that first derivatives of solutions to such problems $\mathrm{P}_{N}$ lie in a CEF.

For each problem $\mathrm{P}_{N}, N \geq N_{1}$, its minimizers $u_{N}$ cannot have derivatives whose modulus exceeds $N_{1}$ for all $x \in[a, b]$. Otherwise we would have $F\left[u_{N}\right]>F[p]$ where $p$ is an admissible linear function. Let $\delta=\delta(x, y)$ be a modulus associated with the CEF. Thus for $|a-b| \leq \eta_{0} \leq \delta\left(N_{1}, 1\right)$ we conclude that each minimizer $u_{N}$ satisfies

$$
\left|\dot{u}_{N}(t)\right| \leq N_{1}+1 \quad \forall t \in[a, b] .
$$

Clearly (3.3) implies that it suffices to utilize the modulus $\delta(N, y)$ associated with $N=N_{1}+1$. Therefore we have proved that for a fixed $M>0, \varepsilon \leq \varepsilon_{0}$ and $(a, A) \in K$ and for all $\eta \leq \eta_{0}=\delta\left(N_{1}, 1\right)$ the problems $\mathrm{P}_{N}$ have solutions in $W^{1, \infty}$ with derivatives bounded in modulus by $N_{1}+1$. In view of Theorem 1.2, Corollary 2 of [S1] we deduce that the $\left\{\dot{u}_{N}\right\}$ are bounded in $C^{0, \gamma}$-norm for some $\gamma>0$. Note that we have actually proved that for a fixed $M>0, \varepsilon<\varepsilon_{0}$ and $(a, A) \in K$ the minimization problem over all Lipschitz functions with graphs in $\mathbf{G}(a, A ; \varepsilon)$ has a solution $u_{0}$ provided that $|a-b| \leq \eta \leq \eta_{0},|A-B| /|a-b| \leq M$. Indeed $u_{0}$ can be chosen as the limit of a sequence of solutions $u_{N_{m}}$ to $\mathrm{P}_{N_{m}}$ converging in $C^{1}$-norm. Moreover, all such minimizers are equibounded in $C^{1, \gamma}$ norm for some $\gamma<0$ (uniformly with respect to $\left.(a, A) \in K, \varepsilon \leq \varepsilon_{0}, \eta \leq \eta_{0}\right)$.

(2) We prove the assertion of the theorem first for $L$ with superlinear growth: $L(t, u, v) \geq \vartheta(|v|)>0$, where $\vartheta(\rho) / \rho \rightarrow \infty$ as $\rho \rightarrow \infty, \vartheta$ is an increasing function on $[0, \infty)$ and $\vartheta^{\prime \prime}>0$ everywhere.

Let $\bar{u}$ be an admissible function with essentially unbounded derivative which satisfies $(*) F[\bar{u}] \leq F\left[u_{0}\right]$, where $u_{0}$ is a minimizer over all Lipschitz functions (so $u_{0}$ satisfies $\left.(3.3)\right)$. For $(t, u) \in \mathbf{G}(a, A ; \varepsilon)((a, A)$ being fixed) we will construct a function $\widetilde{L} \leq L$ satisfying the following requirements:

$$
\begin{array}{r}
\widetilde{L}(t, u, v)=L(t, u, v) \quad \text { for }|v| \leq N_{1}+1, \\
\widetilde{L}(t, u, v)=\vartheta(|v|) / 2 \quad \text { for }|v| \text { sufficiently large. }
\end{array}
$$

For the given $(a, A)$ put

$$
\begin{aligned}
M_{0} & =\sup \left\{L(t, u, v)|(t, u) \in \mathbf{G}(a, A ; \varepsilon),| v \mid \leq N_{1}+1\right\}, \\
K_{0} & =\sup \left\{|l|\left|l \in \partial_{v} L(t, u, v),(t, u) \in \mathbf{G}(a, A ; \varepsilon),\right| v \mid \leq N_{1}+1\right\}, \\
N_{2} & =\min \left\{\rho \geq N_{1}+1 \mid \vartheta(\rho) / 2 \geq M_{0}+K_{0}\left(\rho+N_{1}+1\right)\right\} .
\end{aligned}
$$

For $(t, u) \in \mathbf{G}(a, A ; \varepsilon)$ we define

$$
\begin{aligned}
\bar{L}(t, u, v) & =L(t, u, v), \quad|v| \leq N_{2}, \\
& =\infty, \quad|v|>N_{2} .
\end{aligned}
$$

We also put $\bar{\vartheta}(\rho)=\max \left\{\vartheta(\rho), \vartheta\left(N_{2}\right)\right\}$. Note that for $|v| \geq N_{2}$

$$
\begin{array}{r}
L(t, u, v)>\vartheta(|v|) / 2 \geq \max \left\{L\left(t, u, v_{0}\right)+\left\langle l, v-v_{0}\right\rangle \mid(t, u) \in \mathbf{G}(a, A ; \varepsilon),\right. \\
\left.\left|v_{0}\right| \leq N_{1}+1,\left.l \in \partial L(t, u, \cdot)\right|_{v_{0}}\right\} .
\end{array}
$$


Let $\widetilde{L}(t, u, v)$ be the convexification with respect to $v$ of the function $f(t, u, v)=$ $\min \{\bar{L}(t, u, v), \bar{\vartheta}(|v|) / 2\}$. We now check that $\widetilde{L} \leq L$ as well as the validity of (3.4) and (3.5). Since $f \leq \bar{L} \leq L$ for $|v| \leq N_{2}$, while $f \leq \vartheta(|v|) / 2 \leq L$ for $|v| \geq N_{2}$, the relation $\widetilde{L} \leq L$ is clear. Now suppose $\left|v_{0}\right|<N_{1}+1$ and take $\left.l \in \partial L(t, u, \cdot)\right|_{v_{0}}$. Then $f\left(t, u, v_{0}\right)=L\left(t, u, v_{0}\right)$. Moreover for all $v f(t, u, v) \geq$ $L\left(t, u, v_{0}\right)+\left\langle l, v-v_{0}\right\rangle:=g_{v_{0}}(t, u, v)$. This follows where $f(t, u, v)=L(t, u, v)$ from the convexity of $L$ in $v$, while it follows where $f(t, u, v)=\bar{\vartheta}(|v|) / 2$ from the definition of $N_{2}$. Hence (3.4) follows for $\widetilde{L}\left[f\left(t, u, v_{0}\right) \geq \widetilde{L}\left(t, u, v_{0}\right) \geq g_{v_{0}}\right.$, since $g_{v_{0}}$ is affine and $\left.f\left(t, u, v_{0}\right)=g_{v_{0}}\left(t, u, v_{0}\right)\right]$. Similarly, (3.5) holds for $\widetilde{L}$ because $f(t, u, v)=\vartheta(|v|) / 2$ for $|v|>N_{2}(f \geq \widetilde{L} \geq \vartheta / 2$, and $\vartheta(|v|)$ is convex). Thus $\widetilde{L}(t, u, v)=\vartheta(|v|) / 2$ for such $v$.

Moreover $\widetilde{L}(t, u, v)$ is Hölder continuous on compact subsets of $(t, u, v)$ variables. In fact, by the Ekeland lemma [ET, Ch. 9, Lemma 3.2]

$$
\widetilde{L}(t, u, v)=\min \left\{\sum_{1 \leq i \leq n+1} c_{i} f\left(t, u, v_{i}\right): c_{i} \geq 0, \sum_{1 \leq i \leq n+1} c_{i}=1, \sum_{1 \leq i \leq n+1} c_{i} v_{i}=v\right\}
$$

Moreover, in view of the superlinear growth with respect to $v$, for each $M>0$ there exists an $M_{1}>0$ such that if $|v| \leq M$ then all $v_{i}$ can be chosen to satisfy the inequality $\left|v_{i}\right| \leq M_{1}$. This property implies the Hölder continuity of $\widetilde{L}$.

Now it can happen that $\widetilde{L}$ does not satisfy the local ellipticity condition in (H1). In this case we consider the integrands $L_{\nu}=\widetilde{L}+\nu \max \left\{\vartheta(|v|)-\vartheta\left(N_{1}+1\right), 0\right\}$. By (*) $F_{\nu}[\bar{u}]<F_{\nu}\left[u_{0}\right]$ for all sufficiently small $\nu$, and $\vartheta^{\prime \prime}>0$ implies the integrands $L_{\nu}$ satisfy (H1) for $\nu>0$. In view of (3.2)-(3.4) and the choice of $|b-a|$ the function $u_{0}$ is a solution for the problems with integrands $L_{\nu}$ over the Lipschitz functions. On the other hand, in view of (3.5) and Vallée-Poussin's criterion there exists a sequence $\left\{u_{m}\right\} \subset W^{1, \infty}$ for which $\left\|u_{m}-\bar{u}\right\|_{W^{1,1}} \rightarrow 0$ and $F_{\nu}\left[u_{m}\right] \rightarrow F_{\nu}(\bar{u})$. This is a contradiction.

(3) Now consider the case in which $L$ does not have superlinear growth. Here we can reduce the situation to the case of at least linear growth in $v$. Let $F[w]<F\left[u_{0}\right]$ for some admissible $w \in W^{1,1}$ with essentially unbounded derivative. Then for some increasing function $\vartheta \in C^{2}([0, \infty))$ with superlinear growth and positive second derivative we have $\int_{a}^{b} \vartheta(|\dot{w}(t)|) d t<\infty$. Therefore for all sufficiently small $\nu>0 F_{\nu}[w]<F_{\nu}\left[u_{0}\right]$, where $F_{\nu}$ is the functional with integrand $L_{\nu}=L+$ $\nu \max \left\{\vartheta(|v|)-\vartheta\left(N_{2}\right), 0\right\}$. However, $u_{0}$ is a minimizer for all integrands $L_{\nu}$ in view of the relation $L_{\nu}=L_{0}$ for $|v| \leq N_{1}+1$. It follows from (2) that this leads to a contradiction. Thus we have proved that solutions in $W^{1, \infty}$ are also solutions in $W^{1,1}$.

Now we will prove that all solutions in $W^{1,1}$ are Lipschitz. Let $w$ be a solution in $W^{1,1}$. Put $k\left(t_{1}, t_{2}\right)=\left(w\left(t_{2}\right)-w\left(t_{1}\right)\right) /\left(t_{2}-t_{1}\right)$. Suppose that for sequences $t_{1}^{m}, t_{2}^{m} \rightarrow t_{0}\left(t_{1}^{m}<t_{0}<t_{2}^{m}\right) k\left(t_{1}^{m}, t_{0}\right) \rightarrow k^{-}, k\left(t_{0}, t_{2}^{m}\right) \rightarrow k^{+}$, with $\left|k^{+}\right|,\left|k^{-}\right|<\infty$. It follows by contradiction that $k^{-}=k^{+}$. Otherwise we could replace $w$ by minimizers $u^{-}, u^{+}$over Lipschitz functions having the same boundary conditions as $w$ on the intervals $\left[t_{1}^{m}, t_{0}\right]$ and $\left[t_{0}, t_{2}^{m}\right]$, respectively. In view of the results in (1), (2) and the boundedness of $k^{-}$and $k^{+}, u^{-}$and $u^{+}$are also bounded in $C^{1}$-norm for sufficiently large $m$. In view of the equicontinuity of their derivatives, $\dot{u}^{-}\left(t_{0}\right) \neq \dot{u}^{+}\left(t_{0}\right)$ for 
these $m$. But (2) implies that the function

$$
\begin{aligned}
w_{1} & =u^{+}(t), & t \in\left[t_{1}^{m}, t_{0}\right], \\
& =u^{-}(t), & t \in\left[t_{0}, t_{2}^{m}\right],
\end{aligned}
$$

is a solution in $W^{1, \infty}$. This contradicts the regularity proved in (2) for such solutions. Next suppose that only one of $k^{-}$and $k^{+}$can be taken to be finite, say $\left|k^{-}\right|<\infty$. We again arrive at a contradiction in similar fashion. We can choose $\tau^{m} \rightarrow t_{0}-0$ for which $k\left(\tau^{m}, t_{0}\right) \rightarrow k^{-}$. Moreover there exist sequences $\tau_{1}^{m} \rightarrow t_{0}-0$, $\tau_{2}^{m} \rightarrow t_{0}+0$ for which $k\left(\tau^{m}, \tau_{1}^{m}\right) \rightarrow k^{-}, k\left(\tau_{1}^{m}, \tau_{2}^{m}\right) \rightarrow k_{0}$ such that $\left|k^{-}\right|<\left|k_{0}\right|<\infty$. Now consider minimizers $u^{-}, u^{+}$over the class of Lipschitz functions with boundary conditions given by the values of $w$ at $\tau^{m}, \tau_{1}^{m}$ and $\tau_{1}^{m}, \tau_{2}^{m}$, respectively. For all sufficiently large $m$ such minimizers exist, and their derivatives are equicontinuous functions by the results in (1) and (2). Therefore $\dot{u}^{+}\left(\tau_{1}^{m}\right) \neq \dot{u}^{-}\left(\tau_{1}^{m}\right)$ for $m$ sufficiently large. But the function which is given by $u^{-}$on $\left[\tau^{m}, \tau_{1}^{m}\right]$ and $u^{+}$on $\left[\tau_{1}^{m}, \tau_{2}^{m}\right]$ is a minimizer over Lipschitz functions according to (2). This contradicts the regularity properties obtained for such minimizers.

We are now able to complete the proof of regularity for $w$. In view of what was shown above, $w$ has a finite derivative at each point where at least one of $k^{-}$and $k^{+}$ is finite. Moreover the derivative is bounded and continuous in some neighborhood of each point of this type. Namely, if $t_{0}$ is such a point then $k^{-}=k^{+}=\dot{w}\left(t_{0}\right)$. Therefore for each $t_{1}, t_{2}$ sufficiently near $t_{0}$ with $t_{1}<t_{0}<t_{2}$ we can replace $w$ on $\left[t_{1}, t_{0}\right],\left[t_{0}, t_{2}\right]$ by respective minimizers $u^{-}, u^{+}$over $W^{1, \infty}$, while maintaining the property that $w$ is a minimizer over $W^{1,1}$. Hence $\dot{u}^{-}\left(t_{1}\right)=\dot{w}\left(t_{1}\right), \dot{u}^{+}\left(t_{2}\right)=\dot{w}\left(t_{2}\right)$. In view of the equicontinuity result obtained in (1) for minimizers over $W^{1, \infty}$ we conclude that $\dot{w}\left(t_{1}\right), \dot{w}\left(t_{2}\right) \rightarrow \dot{w}\left(t_{0}\right)$ as $t_{1}, t_{2} \rightarrow t_{0}$.

Now let $t_{0}$ be a point where $\dot{w}\left(t_{0}\right)$ exists with $\left|\dot{w}\left(t_{0}\right)\right| \leq N_{1}$, with $N_{1}$ as in (1). Examine $J_{0}=\bigcup J$, where $t_{0} \in J$ and $\dot{w}$ is bounded on the segment $J$. In view of (1) $|\dot{w}(t)| \leq N_{1}+1$ everywhere on $J_{0}$. Hence in view of what was proved above $J_{0}$ can be extended to the interval $[a, b]$. This complete the proof.

Proof of Theorem 2. Let $u$ be one of the functions under consideration, namely a minimizer of (1) whose graph lies in $K$, where $K$ is a compact set in $\mathbb{R} \times \mathbb{R}^{n}$. Let $t_{0}$ be a point in the domain of $u$ such that

$$
\liminf _{t_{1}, t_{2} \rightarrow t_{0}, t_{1}<t_{0}<t_{2}}\left|\left(u\left(t_{2}\right)-u\left(t_{1}\right)\right) /\left(t_{2}-t_{1}\right)\right|<\infty .
$$

In view of Theorem 1 we deduce that $u$ is $C^{1}$-regular in some neighborhood of $t_{0}$. Thus $u \in C^{1}(\Omega)$ for an open set $\Omega$ of full measure in its domain. Moreover, at all other points $t$ in its domain $u$ satisfies

$$
\liminf _{t_{1}, t_{2} \rightarrow t, t_{1}<t<t_{2}}\left|\left(u\left(t_{2}\right)-u\left(t_{1}\right)\right) /\left(t_{2}-t_{1}\right)\right|=\infty .
$$

In order to prove that the derivatives $\{\dot{u}\}$ of these functions form a CEF we first show that for any $N>0$ and $\varepsilon \in(0,1 / 2)$ there exists $\bar{\eta}>0$ such that if $u$ is any one of the functions under consideration and $\left|\dot{u}\left(t_{0}\right)\right|<N$ then $\left|\dot{u}(t)-\dot{u}\left(t_{0}\right)\right| \leq \varepsilon$ whenever $\left|t-t_{0}\right| \leq \bar{\eta}$.

Put $M=N+1$, let $\varepsilon_{0}, \eta_{0}$ denote the values associated in Theorem 1 to $K$ and $M$ and let $\nu$ denote the modulus of equicontinuity for the functions $\{\dot{u}\}$ furnished by Theorem 1 . We select $\bar{\eta}$ subject to the following conditions:

$$
\nu(\bar{\eta}) \leq \varepsilon / 2, \quad \varepsilon_{0}>M \bar{\eta} \quad\left[\bar{\eta}<\min \left\{\varepsilon_{0}, \eta_{0}\right\}\right] .
$$


We now prove by contradiction that $\left|t-t_{0}\right| \leq \bar{\eta}$ implies $\left|\dot{u}(t)-\dot{u}\left(t_{0}\right)\right| \leq \varepsilon$. Otherwise, there is some $t \in J=\left(t_{0}-\bar{\eta}, t_{0}+\bar{\eta}\right)$ for which $\left|\dot{u}(t)-\dot{u}\left(t_{0}\right)\right|>\varepsilon$. Hence if $t>t_{0}$ there exists $t_{1} \in J$ such that $|\dot{u}(t)| \leq M$ for all $t \in\left[t_{0}, t_{1}\right]$ and $\left|\dot{u}\left(t_{1}\right)-\dot{u}\left(t_{0}\right)\right|=\varepsilon$ [if $t<t_{0}$ the argument is similar]. Now by the second inequality in (3.8) the graph of $u$ over $\left[t_{0}, t_{1}\right]$ lies in

$$
\mathbf{G}\left(t_{0}, u\left(t_{0}\right) ; \varepsilon_{0}\right)=\left\{(t, u)|| t-t_{0}\left|\leq \varepsilon_{0},\right| u^{i}-u^{i}\left(t_{0}\right) \mid \leq \varepsilon_{0}, 1 \leq i \leq n\right\} .
$$

In view of Theorem 1 and the first inequality in (3.8) we have $\left|\dot{u}\left(t_{1}\right)-\dot{u}\left(t_{0}\right)\right| \leq$ $\nu\left(\left|t_{1}-t_{0}\right|\right) \leq \varepsilon / 2$, which gives a contradiction. Hence $\left|\dot{u}(t)-\dot{u}\left(t_{0}\right)\right| \leq \varepsilon$ for all $t \in J$. To complete the proof we need to show that if $u$ is one of the functions under consideration and $t_{0} \notin \Omega$, where $\Omega$ is the set of regularity for $u$, then $\lim _{t \rightarrow t_{0}, t \in \Omega}|\dot{u}(t)|=\infty$. Note however that if there exists a sequence $\left\{t_{n}\right\} \subset \Omega$ satisfying $t_{n} \rightarrow t_{0}$ such that $\left\{\left|\dot{u}\left(t_{n}\right)\right|\right\}$ is bounded then $\Omega$ includes the $\eta$-neighborhoods of $\left\{t_{n}\right\}$ for some $\eta$, and $t_{0}$ necessarily lies in $\Omega$ as well. This completes the argument.

\section{Proof of Theorem 3}

Proof of Theorem 3. For (1) with given boundary conditions $\left(a_{0}, A_{0}, b_{0}, B_{0}\right)$ the superlinear growth condition ensures that the graphs of the set $U$ of all minimizers lies in a compact subset $K$ of $\mathbb{R} \times \mathbb{R}^{n}$. Hence by Theorem 2 the set $V=\left\{u^{\prime} \mid u \in U\right\}$ is a CEF.

1) Now suppose that all $u$ in $U$ have bounded derivatives at $a_{0}$ and $b_{0}$. Then there exists $M>0$ such that the derivatives at $a_{0}$ and $b_{0}$ of all $u$ in $U$ are bounded in modulus by $M$. Otherwise we could select a sequence $\left\{u_{n}\right\} \subset U$ for which $\left|\dot{u}_{n}\left(b_{0}\right)\right| \rightarrow \infty$ [the case $\left|\dot{u}_{n}\left(a_{0}\right)\right| \rightarrow \infty$ can be treated analogously]. Furthermore we can suppose by going to a subsequence that $\left\{u_{n}\right\}$ converges uniformly to some function $u_{0}$ on $\left[a_{0}, b_{0}\right]$. Then $u_{0}$ is also a minimizer for (1), by Tonelli's lower semicontinuity theorem or Lemma 2.1. By hypothesis $\left|\dot{u}_{0}\left(b_{0}\right)\right|<\infty$, so that $b_{0}$ is a point of the relatively open subset $\Omega\left(\dot{u}_{0}\right)$. Consequently by Lemma 2.2 there is an interval $\left[b_{0}-\eta, b_{0}\right]$ such that $\left\|\dot{u}_{n}-\dot{u}_{0}\right\|_{C\left[b_{0}-\eta, b_{0}\right]} \rightarrow 0$, which contradicts the assumption $\left|\dot{u}_{n}\left(b_{0}\right)\right| \rightarrow \infty$.

2) We now show that the value function $S$ defined in (2) is continuous at $\left(a_{0}, A_{0}, b_{0}, B_{0}\right) . S$ is lower semicontinuous at $\left(a_{0}, A_{0}, b_{0}, B_{0}\right)$ by virtue of Tonelli's theorem or Lemma 2.1. Now let $\left(a_{n}, A_{n}, b_{n}, B_{n}\right) \rightarrow\left(a_{0}, A_{0}, b_{0}, B_{0}\right)$ and let $u_{n}$ denote a corresponding minimizer for each $n \geq 0$. In view of the property $\left|\dot{u}_{0}\left(a_{0}\right)\right|$, $\left|\dot{u}_{0}\left(b_{0}\right)\right| \leq M$ we can modify $u_{0}$ to produce an admissible function $\tilde{u}_{n}$ for the $n$th problem (by taking $\tilde{u}_{n}$ to be linear in appropriate neighborhoods of $a_{n}$ and $b_{n}$ with derivatives equibounded in modulus on these neighborhoods) in such a way that $F_{\left(a_{n}, b_{n}\right)}\left[\tilde{u}_{n}\right] \rightarrow F_{\left(a_{0}, b_{0}\right)}\left[u_{0}\right]$ [this construction will be made explicit in 3)]. Thus

$$
S\left(a_{0}, A_{0}, b_{0}, B_{0}\right)=F\left[u_{0}\right] \geq \underset{n}{\lim \sup } S\left(a_{n}, A_{n}, b_{n}, B_{n}\right),
$$

which completes the proof of continuity.

3) Next we prove that for all boundary conditions $(a, A, b, B)$ sufficiently near to $\left(a_{0}, A_{0}, b_{0}, B_{0}\right)$ the corresponding minimizers will have derivatives on neighborhoods of $a$ and $b$ which are bounded in modulus by $M+1$. By Theorem 2 the set $V=\{\dot{u}\}$ of derivatives of all such minimizers is a CEF, so that the above assertion is an immediate consequence of 1 ), Lemma 2.2 and 2). The continuity of $S$ implies that uniform limits of solutions $u_{n}$ for problems with boundary conditions 
$\left(a_{n}, A_{n}, b_{n}, B_{n}\right) \rightarrow\left(a_{0}, A_{0}, b_{0}, B_{0}\right)$ are solutions for the problem with boundary conditions $\left(a_{0}, A_{0}, b_{0}, B_{0}\right)$.

This fact permits us to prove the Lipschitz continuity of $S$ at $\left(a_{0}, A_{0}, b_{0}, B_{0}\right)$. Namely, with $u$ a minimizer for $(a, A, b, B)$ and $u_{0}$ a minimizer for $\left(a_{0}, A_{0}, b_{0}, B_{0}\right)$, put $\tilde{a}=\max \left\{a_{0}, a\right\}, \tilde{b}=\min \left\{b_{0}, b\right\}$. Then

$$
\begin{aligned}
\left|\int_{a}^{b} L(t, u(t), \dot{u}(t)) d t-\int_{a_{0}}^{b_{0}} L\left(t, u_{0}(t), \dot{u}_{0}(t)\right) d t\right| \\
\leq\left|\int_{\tilde{a}}^{\tilde{b}}\left[L(t, u(t), \dot{u}(t))-L\left(t, u_{0}(t), \dot{u}_{0}(t)\right)\right] d t\right| \\
\quad+\int_{a}^{\tilde{a}}|L(t, u(t), \dot{u}(t))| d t+\int_{\tilde{b}}^{b}|L(t, u(t), \dot{u}(t))| d t \\
\quad+\int_{a_{0}}^{\tilde{a}}\left|L\left(t, u_{0}(t), \dot{u}_{0}(t)\right)\right| d t+\int_{\tilde{b}}^{b_{0}}\left|L\left(t, u_{0}(t), \dot{u}_{0}(t)\right)\right| d t \\
\leq\left|\int_{\tilde{b}}^{\tilde{b}}\left[L(t, u(t), \dot{u}(t))-L\left(t, u_{0}(t), \dot{u}_{0}(t)\right)\right] d t\right| \\
\quad+c(|a-\tilde{a}|+|b-\tilde{b}|),
\end{aligned}
$$

where $c=\max \{L(t, u, v)|(t, u) \in K| v \mid, \leq M+1\}$ and $K$ is a compact set including the graphs of all minimizers under consideration. In particular, if

$$
\left|a-a_{0}\right|+\left|b-b_{0}\right|+\left|A-A_{0}\right|+\left|B-B_{0}\right| \leq \eta,
$$

then

$$
\begin{aligned}
& \left|\int_{a}^{b} L(t, u(t), \dot{u}(t)) d t-\int_{a_{0}}^{b_{0}} L\left(t, u_{0}(t), \dot{u}_{0}(t)\right) d t\right| \\
& \quad \leq\left|\int_{\tilde{a}}^{\tilde{b}}\left[L(t, u(t), \dot{u}(t))-L\left(t, u_{0}(t), \dot{u}(t)\right)\right] d t\right|+c \eta, \\
& \left|u(\tilde{a})-u_{0}(\tilde{a})\right|+\left|u(\tilde{b})-u_{0}(\tilde{b})\right| \leq \eta+2(M+1) \eta .
\end{aligned}
$$

We will use (4.2) to appraise $\left|S\left(a_{n}, A_{n}, b_{n}, B_{n}\right)-S\left(a_{0}, A_{0}, b_{0}, B_{0}\right)\right|$ where $\left(a_{n}, A_{n}\right.$, $\left.b_{n}, B_{n}\right) \rightarrow\left(a_{0}, A_{0}, b_{0}, B_{0}\right)$ and $(*)$ is satisfied. Letting $\left\{u_{n}\right\}_{n \geq 0}$ denote minimizers, as above, it is clear that the restrictions of $u_{n}, n \geq 0$, to any interval $J \subset\left[a_{n}, b_{n}\right]$ are minimizers for the associated boundary data at the endpoints of $J$. Now suppose for a given $n>0$

$$
\begin{aligned}
F_{\left(\tilde{a}_{n}, \tilde{b}_{n}\right)}\left[u_{n}\right] & =S\left(\tilde{a}_{n}, u_{n}\left(\tilde{a}_{n}\right), \tilde{b}_{n}, u_{n}\left(\tilde{b}_{n}\right)\right) \\
& \geq S\left(\tilde{a}_{n}, u_{0}\left(\tilde{a}_{n}\right), \tilde{b}_{n}, u_{0}\left(\tilde{b}_{n}\right)\right)=F_{\left(\tilde{a}_{n}, \tilde{b}_{n}\right)}\left[u_{0}\right] .
\end{aligned}
$$

We proceed to construct an admissible function $\tilde{u}_{n}$ on $\left[\tilde{a}_{n}, \tilde{b}_{n}\right]$ (for $\eta$ sufficiently small) as follows:

$$
\tilde{u}_{n}(t)=u_{0}(t) \quad \text { for } t \in\left[\tilde{a}_{n}+\eta, \tilde{b}_{n}-\eta\right]
$$

so that

$$
\left|\dot{u}_{n}(t)\right|,\left|\dot{u}_{0}(t)\right| \leq M+2 \quad \text { for } t \in\left[\tilde{a}_{n}, \tilde{a}_{n}+\eta\right] \quad \text { and } \quad\left[\tilde{b}_{n}-\eta, \tilde{b}_{n}\right] .
$$


$\tilde{u}_{n}\left(\tilde{a}_{n}\right)=u_{n}\left(\tilde{a}_{n}\right), \tilde{u}_{n}\left(\tilde{b}_{n}\right)=u_{n}\left(\tilde{b}_{n}\right), \tilde{u}_{n}$ has constant derivative on $\left[\tilde{a}_{n}, \tilde{a}_{n}+\eta\right]$ and on $\left[\tilde{b}_{n}-\eta, \tilde{b}_{n}\right]$. Thus by $(4.2)\left|\tilde{u}_{n}^{\prime}(t)\right| \leq 4 M+8$ on these intervals. Then by virtue of the boundedness of $L$ on bounded sets in $\mathbb{R} \times \mathbb{R}^{n} \times \mathbb{R}^{n}$

$$
\begin{aligned}
0 \leq & F_{\left(\tilde{a}_{n}, \tilde{b}_{n}\right)}\left[\tilde{u}_{n}\right]-F_{\left(\tilde{a}_{n}, \tilde{b}_{n}\right)}\left[u_{0}\right] \leq \int_{\tilde{a}_{n}}^{\tilde{a}_{n}+\eta}\left|L\left(t, \tilde{u}_{n}(t), \tilde{u}_{n}^{\prime}(t)\right)\right| d t \\
& +\int_{\tilde{b}_{n}-\eta}^{\tilde{b}_{n}}\left|L\left(t, \tilde{u}_{n}(t), \tilde{u}_{n}^{\prime}(t)\right)\right| d t \leq c_{2} \eta .
\end{aligned}
$$

Since $F_{\left(\tilde{a}_{n}, \tilde{b}_{n}\right)}\left[u_{n}\right] \leq F_{\left(\tilde{a}_{n}, \tilde{b}_{n}\right)}\left[\tilde{u}_{n}\right]$, we conclude by (4.2) that

$$
\left|S\left(a_{n}, A_{n}, b_{n}, B_{n}\right)-S\left(a_{0}, A_{0}, b_{0}, B_{0}\right)\right| \leq\left(c+c_{2}\right) \eta .
$$

An analogous approach applies for those values of $n$ for which

$$
\begin{aligned}
F_{\left(\tilde{a}_{n}, \tilde{b}_{n}\right)}\left[u_{n}\right] & =S\left(\tilde{a}_{n}, u_{n}\left(\tilde{a}_{n}\right), \tilde{b}_{n}, u_{n}\left(\tilde{b}_{n}\right)\right) \\
& \leq S\left(\tilde{a}_{n}, u_{0}\left(\tilde{a}_{n}\right), \tilde{b}_{n}, u_{0}\left(\tilde{b}_{n}\right)\right)=F_{\left(\tilde{a}_{n}, \tilde{b}_{n}\right)}\left[u_{0}\right] .
\end{aligned}
$$

In such cases the construction of $\tilde{u}_{n}$ is given as:

$$
\begin{aligned}
& \tilde{u}_{n}(t)=u_{n}(t) \text { for } t \in\left[\tilde{a}_{n}+\eta, \tilde{b}_{n}-\eta\right], \text { for } \eta \text { so small that } \\
& \left|\dot{u}_{n}(t)\right|,\left|\dot{u}_{0}(t)\right| \leq M+2 \text { for } t \in\left[\tilde{a}_{n}, \tilde{a}_{n}+\eta\right] \text { and }\left[\tilde{b}_{n}-\eta, \tilde{b}_{n}\right], \tilde{u}_{n}\left(\tilde{a}_{n}\right)= \\
& u_{0}\left(\tilde{a}_{n}\right), u_{n}\left(\tilde{b}_{n}\right)=u_{0}\left(\tilde{b}_{n}\right), \tilde{u}_{n} \text { has constant derivative on }\left[\tilde{a}_{n}, \tilde{a}_{n}+\eta\right] \\
& \text { and on }\left[\tilde{b}_{n}-\eta, \tilde{b}_{n}\right] .
\end{aligned}
$$

Thus by (4.2) $\left|\tilde{u}_{n}^{\prime}(t)\right| \leq 4 M+8$ on these intervals. Thus we obtain

$$
\begin{aligned}
0 \leq & F_{\left(\tilde{a}_{n}, \tilde{b}_{n}\right)}\left[u_{0}\right]-F_{\left(\tilde{a}_{n}, \tilde{b}_{n}\right)}\left[\tilde{u}_{n}\right] \\
\leq & \int_{\tilde{a}_{n}}^{\tilde{a}_{n}+\eta}\left|L\left(t, \tilde{u}_{n}(t), \tilde{u}_{n}^{\prime}(t)\right)\right| d t \\
& +\int_{\tilde{b}_{n}-\eta}^{\tilde{b}_{n}}\left|L\left(t, \tilde{u}_{n}(t), \tilde{u}_{n}^{\prime}(t)\right)\right| d t \leq c_{2} \eta,
\end{aligned}
$$

from which (4.6) again follows.

4) Finally, we consider the case in which problem (1) with boundary conditions $\left(a_{0}, A_{0}, b_{0}, B_{0}\right)$ has a minimizer possessing an infinite derivative at $b_{0}$ [the case with an infinite derivative at $a_{0}$ can be reduced to this one]. Let $u_{0}$ be a minimizer for (1) such that

$$
\lim _{t \rightarrow b_{0}-0}\left|u_{0}\left(b_{0}\right)-u_{0}(t)\right| /\left|b_{0}-t\right|=\infty .
$$

We prove that $S\left(a_{0}, A_{0}, b, B_{0}\right)$ cannot be Lipschitz at $b_{0}$. For fixed $b>b_{0}$ let us denote by $t_{0}(b)$ the largest value of $t<b_{0}$ for which $\left|\left(B_{0}-u_{0}(t)\right) /(b-t)\right|=1$. It is clear that $t_{0}(b)$ is defined by all $b$ sufficiently close to $b_{0}$ from the right and that $\left(b-t_{0}(b)\right) /\left(b-b_{0}\right) \rightarrow 1$ as $b \rightarrow b_{0^{+}}$. We now define an admissible function $u_{b}$ for $\left(a_{0}, A_{0}, b, B_{0}\right)$ by

$$
\begin{aligned}
u_{b}(t) & =u_{0}(t) & & \text { for } t \in\left[a_{0}, t_{0}(b)\right] \\
& =C_{b}\left(t-t_{0}(b)\right)+u_{0}\left(t_{0}(b)\right) & & \text { for } t \in\left(t_{0}(b), b\right],
\end{aligned}
$$


with $\left|C_{b}\right|=1$. Using the hypothesis concerning a convex function $\vartheta$ with superlinear growth, we obtain the following appraisal:

$$
\begin{aligned}
& S\left(a_{0}, A_{0}, b_{0}, B_{0}\right)-S\left(a_{0}, A_{0}, b, B_{0}\right) \\
& \quad \geq \int_{t_{0}(b)}^{b_{0}} \vartheta\left(u_{0}^{\prime}(t)\right) d t-\int_{t_{0}(b)}^{b} L\left(t, u_{b}(t), u_{b}^{\prime}(t)\right) d t \\
& \quad \geq\left(b_{0}-t_{0}(b)\right) \vartheta\left(\left[B_{0}-u_{0}\left(t_{0}(b)\right)\right] /\left[b_{0}-t_{0}(b)\right]\right)-K\left(b-t_{0}(b)\right) .
\end{aligned}
$$

Hence

$\left[S\left(a_{0}, A_{0}, b_{0}, B_{0}\right)-S\left(a_{0}, A_{0}, b, B_{0}\right)\right] /\left(b-b_{0}\right)$

$$
\geq \vartheta\left(\left[B_{0}-u_{0}\left(t_{0}(b)\right)\right] /\left[b_{0}-t_{0}(b)\right]\right)\left(b_{0}-t_{0}(b)\right) /\left(b-b_{0}\right)-K\left(b-t_{0}(b)\right) /\left(b-b_{0}\right) .
$$

[Here $\left|B_{0}-u_{0}\left(t_{0}(b)\right)\right| /\left|b-t_{0}(b)\right|=1$ and $\left(b-t_{0}(b)\right) /\left(b-b_{0}\right) \rightarrow 1$ as $b \rightarrow b_{0^{+}}$. ]

Since $B_{0}-u_{0}\left(t_{0}(b)\right) /\left(b-b_{0}\right) \rightarrow 1$ as $b \rightarrow b_{0^{+}}$and $\left|B_{0}-u_{0}\left(t_{0}(b)\right)\right| /\left|b_{0}-t_{0}(b)\right| \rightarrow \infty$, the limit of the difference quotient in (4.8) is $+\infty$ by the superlinear growth of $\vartheta$. This shows that the right derivative of $S\left(a_{0}, A_{0}, b, B_{0}\right)$ at $b_{0}$ is $-\infty$, contradicting the Lipschitz property. This completes the proof of Theorem 3

Remark 1. The proof above actually demonstrates a stronger assertion: regularity of the $\left(a_{0}, A_{0}, b_{0}, B_{0}\right)$ minimizers at $b_{0}$ holds if and only if the function $S\left(a_{0}, A_{0}, b, B_{0}\right)$ is Lipschitz at $b_{0}$. Under the assumptions of Theorem 3 it is also true that problem (1) with boundary conditions $\left(a_{0}, A_{0}, b_{0}, B_{0}\right)$ has all minimizers regular at $b_{0}$ if and only if the function $S\left(a_{0}, A_{0}, b_{0}, B\right)$ is Lipschitz at $B_{0}$. In $\S 5$ we will use these assertions in the proof of Theorem 4 .

Proof. The Lipschitz property of $S\left(a_{0}, A_{0}, b_{0}, B\right)$ at $B_{0}$ is a consequence of the proof of the theorem given above. Let $u_{0}$ be a minimizer for (1) with boundary conditions $\left(a_{0}, A_{0}, b_{0}, B_{0}\right)$ such that

$$
\lim _{t \rightarrow b_{0}-0}\left|u_{0}\left(b_{0}\right)-u_{0}(t)\right| /\left|b_{0}-t\right|=\infty .
$$

(It again suffices to consider the case in which problem (1) with boundary conditions $\left(a_{0}, A_{0}, b_{0}, B_{0}\right)$ has a minimizer possessing an infinite derivative at $b_{0}$.) We prove that $S\left(a_{0}, A_{0}, b_{0}, B\right)$ does not have the Lipschitz property at $B_{0}$. For $\eta>0$ define an admissible function $u_{\eta}$ for problem (1) with boundary condition $\left(a_{0}, A_{0}, b_{0}, B_{\eta}\right)$ where $B_{\eta}=u_{0}\left(b_{0}-\eta\right)$ via:

$$
\begin{aligned}
u_{\eta}(t) & =u_{0}(t) & & \text { for } t \in\left[a_{0}, b_{0}-\eta\right] \\
& =u_{0}\left(b_{0}-\eta\right) & & \text { for } t \in\left[b_{0}-\eta, b_{0}\right] .
\end{aligned}
$$

Then for some convex function $\vartheta$ of superlinear growth

$$
\begin{aligned}
& S\left(a_{0}, A_{0}, b_{0}, B_{0}\right)-S\left(a_{0}, A_{0}, b_{0}, B_{\eta}\right) \geq F_{\left(a_{0}, b_{0}\right)}\left[u_{0}\right]-F_{\left(a_{0}, b_{0}\right)}\left[u_{\eta}\right] \\
& \quad \geq \int_{b_{0}-\eta}^{b_{0}} \vartheta\left(\dot{u}_{0}(t)\right) d t-\int_{b_{0}-\eta}^{b_{0}} L\left(t, u_{0}\left(b_{0}-\eta\right), 0\right) d t \\
& \quad \geq \eta \vartheta\left(\left[B_{0}-u_{0}\left(b_{0}-\eta\right)\right] / \eta\right)-c \eta,
\end{aligned}
$$

where $c=\max \{L(t, u, 0) \mid(t, u) \in K\}$. Using the superlinearity of $\vartheta$ and the infinite slope of $u_{0}$ at $b_{0}$ it follows from the above that

$$
\begin{aligned}
& \left|S\left(a_{0}, A_{0}, b_{0}, B_{0}\right)-S\left(a_{0}, A_{0}, b_{0}, B_{\eta}\right)\right| /\left|B_{0}-B_{\eta}\right| \\
& \quad \geq\left(\eta /\left|B_{0}-B_{\eta}\right|\right)\left[\vartheta\left(\left[B_{0}-B_{\eta}\right] / \eta\right)-c\right] \rightarrow \infty \quad \text { as } \eta \rightarrow 0,
\end{aligned}
$$


thereby demonstrating that $S\left(a_{0}, A_{0}, b_{0}, B\right)$ is not Lipschitz at $B_{0}$, as claimed.

Remark 2. Using arguments applied in the proof of Theorem 3 it is not hard to prove that if $K$ is a compact subset of boundary data $(a, A, b, B)$ at each of which $S$ is Lipschitz, then $S$ is uniformly Lipschitz over $K$.

\section{Application of the MAin Result FOR ESTABlishing FUlL REGUlarity UNDER CERTAIN CONDITIONS ON INTEGRANDS}

Theorem 4. Let $L(t, u, v): \mathbb{R} \times \mathbb{R}^{n} \times \mathbb{R}^{n} \rightarrow \mathbb{R}$ satisfy condition (H1) and have superlinear growth. Then any of properties 1)-3) below ensures full regularity of all solutions of problem (1).

1) For each compact set $K$ of $(t, u)$ variables we have

$$
\left|\frac{L\left(t_{1}, u, v\right)-L\left(t_{2}, u, v\right)}{t_{1}-t_{2}}\right| \leq c\left|L\left(t_{1}, u, v\right)\right|+\gamma(t) \quad \text { for some } \gamma \in L_{1} \text {. }
$$

2) For each compact set of $(t, u)$ variables we have

$$
\left|\frac{L\left(t, u_{1}, v\right)-L\left(t, u_{2}, v\right)}{u_{1}-u_{2}}\right| \leq c\left|L\left(t, u_{1}, v\right)\right|+\gamma(t) \quad \text { for some } \gamma \in L_{1} .
$$

3) For each $t \in \mathbb{R}$ the integrand $L(t, u, v)$ is convex in $(u, v)$.

Remark. The integrand $L(t, u, v)$ clearly has property 1$)$ or property 2$)$ if $L$ does not depend on $t$ or $u$, respectively, for all sufficiently large $|v|$.

Proof. (Theorem 4).

1) For some boundary conditions $\left(a_{0}, A_{0}, b_{0}, B_{0}\right)$ suppose a solution has unbounded derivative at some point. It is enough to consider the case

$$
\lim _{t \rightarrow b_{0}-0}\left|\frac{u_{0}\left(b_{0}\right)-u_{0}(t)}{b_{0}-t}\right|=\infty \text {. }
$$

The general situation can be reduced to this one. Let $a_{1} \in\left(a_{0}, b_{0}\right)$ be such that $\left|\dot{u}\left(a_{1}\right)\right|<\infty$. Consider the problem with boundary conditions $\left(a_{1}, A_{1}, b_{0}+\varepsilon, B_{0}\right)$ where $A_{1}=u_{0}\left(a_{1}\right)$, and examine solutions $u_{\varepsilon}$ corresponding to $\varepsilon>0$. We have $\dot{u}_{\varepsilon}\left(a_{1}\right) \rightarrow \dot{u}\left(a_{1}\right)$. Otherwise for some sequence $\varepsilon_{k} \rightarrow+0 \dot{u}_{\varepsilon_{k}}\left(a_{1}\right) \rightarrow A \neq \dot{u}_{0}\left(a_{1}\right)$ and $u_{\varepsilon_{k}}$ converges uniformly to $\tilde{u}_{0}$. In view of Lemmas 2.1 and $2.2, \dot{\tilde{u}}_{0}\left(a_{1}\right)=A$ if $|A|<\infty,\left(\left|\dot{u}\left(a_{1}\right)\right|=\infty\right.$ if $\left.|A|=\infty\right)$. But $S\left(a_{1}, A_{1}, b_{0}+\varepsilon, B_{0}\right) \rightarrow S\left(a_{1}, A_{1}, b_{0}, B_{0}\right)$ as $\varepsilon \rightarrow 0$. Then the function $u$ defined by

$$
u(t)= \begin{cases}u_{0}(t), & a_{0} \leq t \leq a_{1}, \\ \tilde{u}_{0}(t), & a_{1} \leq t \leq b_{0}\end{cases}
$$

is a minimizer for the problem with boundary conditions $\left(a_{0}, A_{0}, b_{0}, B_{0}\right)$. Moreover we have $\dot{u}\left(a_{1}-0\right) \neq \dot{u}\left(a_{1}+0\right)$, but $u_{0}$ satisfies the assertion of Theorem 2 , which yields a contradiction. Therefore we have proved that $\dot{u}_{\varepsilon}\left(a_{1}\right) \rightarrow \dot{u}_{0}\left(a_{1}\right)$ if $\varepsilon \rightarrow+0$. Then in view of Theorem 2

$$
\left|\dot{u}_{\varepsilon}(t)\right| \leq M \quad \text { for } \varepsilon \in\left[0, \varepsilon_{0}\right] \text { and } t \in\left[a_{1}, a_{1}+\delta\right](\delta>0) .
$$

Consider the following admissible functions $u^{\varepsilon}$ associated with the boundary condition $\left(a_{1}, A_{1}, b_{0}, B_{0}\right)$

$$
u^{\varepsilon}(t)= \begin{cases}u_{\varepsilon}(t+\varepsilon), & a_{1}+\varepsilon \leq t \leq b_{0} \\ \dot{u}^{\varepsilon} \equiv c(\varepsilon)=\text { constant }, & a_{1} \leq t \leq a_{1}+\varepsilon\end{cases}
$$


In view of $(* *)|c(\varepsilon)|$ are equibounded for all sufficiently small $\varepsilon>0$. Then

$$
\begin{aligned}
& {\left[S\left(a_{1}, A_{1}, b_{0}+\varepsilon, B_{0}\right)-S\left(a_{1}, A_{1}, b_{0}, B_{0}\right)\right] / \varepsilon} \\
& \quad \geq \frac{F\left[u_{\varepsilon}\right]-F\left[u^{\varepsilon}\right]}{\varepsilon} \geq \frac{\int_{a_{1}}^{b_{0}+\varepsilon} L\left(t, u_{\varepsilon}(t), \dot{u}_{\varepsilon}(t)\right) d t-\int_{a_{1}}^{b_{0}} L\left(t, u^{\varepsilon}(t), \dot{u}^{\varepsilon}(t) d t\right.}{\varepsilon} \\
& \quad \geq M_{1}
\end{aligned}
$$

in view of $(* *)$ and the fact that

$$
\begin{gathered}
\frac{\left|\int_{a_{1}+2 \varepsilon}^{b_{0}+\varepsilon} L\left(t, u_{\varepsilon}(t), \dot{u}_{\varepsilon}(t)\right) d t-\int_{a_{1}+\varepsilon}^{b_{0}} L\left(t, u^{\varepsilon}(t), \dot{u}^{\varepsilon}(t)\right) d t\right|}{\varepsilon} \\
\leq \int_{a_{1}+2 \varepsilon}^{b_{0}+\varepsilon}\left\{\left|L\left(t, u_{\varepsilon}(t), \dot{u}_{\varepsilon}(t)\right)\right|+|\gamma(t)|\right\} d t .
\end{gathered}
$$

As was proved in part 4$)$ of the proof of Theorem $3,\left.S^{\prime}\left(a_{1}, A_{1}, b_{0}+\varepsilon, B_{0}\right)\right|_{\varepsilon=0^{+}}=$ $-\infty$. Thus we have arrived at a contradiction.

2) A proof of assertion 2) of the theorem can be obtained in analogous way. If $\lim _{t \rightarrow b_{0}-0}\left|\frac{u\left(b_{0}\right)-u(t)}{b_{0}-t}\right|=\infty$, then we consider the function $\xi \mapsto S\left(a_{0}, A_{0}, b_{0}, B_{0}+\xi\right)$. If $t_{0} \rightarrow b_{0}-0$ then $B\left(t_{0}\right)=u\left(t_{0}\right) \rightarrow B_{0}$. Let $u_{t_{0}}(t)=u_{0}(t)$ for $a_{0} \leq t \leq t_{0}$ and $u_{t_{0}}(t)=B\left(t_{0}\right)$ for $t_{0} \leq t \leq b_{0}$. Then it was proved in Remark 1 to Theorem 3 that

$$
\frac{S\left(a_{0}, A_{0}, b_{0}, B_{0}\right)-S\left(a_{0}, A_{0}, b_{0}, B\left(t_{0}\right)\right)}{\left|B_{0}-B\left(t_{0}\right)\right|} \rightarrow \infty
$$

as $t_{0} \rightarrow b_{0}-0$ (in this case $\left|B_{0}-B\left(t_{0}\right)\right| \rightarrow 0$ ). But we can also prove that the last expression is bounded by some constant, using arguments similar to those in 1).

3) Here, let $u$ be a minimizer for problem (1) satisfying condition 3) of the theorem. Suppose $u_{0} \notin C^{1}$ and consider the case

$$
\lim _{t \rightarrow b_{0}-0}\left|\frac{u_{0}\left(b_{0}\right)-u_{0}(t)}{b_{0}-t}\right|=\infty \quad \text { (all other cases may be reduced to this one). }
$$

Then, as was proved in Remark 1 to Theorem $3 S\left(a_{0}, A_{0}, b_{0}, B\right)$ is not Lipschitz at $B_{0}$ (here $A_{0}=u_{0}\left(a_{0}\right)$ ). But the function $S$ is convex in $B$ !

Indeed, if $B_{1}, B_{2}$ are sufficiently close to $B_{0}$ and $u_{1}, u_{2}$ are solutions of the corresponding problems then for $\lambda_{1}, \lambda_{2} \geq 0$ such that $\lambda_{1}+\lambda_{2}=1$ we know that $u=\lambda_{1} u_{1}+\lambda_{2} u_{2}$ satisfies the boundary conditions $\left(a_{0}, A_{0}, b_{0}, \lambda_{1} B_{1}+\lambda_{2} B_{2}\right)$.

Therefore, in view of the convexity of $L(t, u, v)$ in $(u, v)$ we deduce that $\lambda_{1} F\left(u_{1}\right)+$ $\lambda_{2} F\left(u_{2}\right) \geq F\left(\lambda_{1} u_{1}+\lambda_{2} u_{2}\right)$. Thus the function $S\left(a_{0}, A_{0}, b_{0}, B\right)$ is convex in $B$ in some neighborhood of $B_{0}$. In this case $S$ is Lipschitz at $B_{0}$, which is a contradiction.

This contradiction permits us to conclude that each minimizer $u_{0}$ is $C^{1}$. This completes the proof.

\section{ACKNOWLEDGMent}

The first-named author wishes to express appreciation for the pleasant environment provided by the Carnegie Mellon Mathematics Department during his stay in Pittsburgh and for the hospitality of his coauthor, of D. Golovaty, of Professor D. Kinderlehrer and other members of the department which made his visit an enjoyable one. 


\section{REFERENCES}

$[\mathrm{BM}]$ J. M. Ball and V. J. Mizel, One-dimensional variational problems whose minimizers do not satisfy the Euler-Lagrange equation, Arch. Rational Mech. Anal. 90 (1985), 325-388. MR 86k:49002

[Bo] O. Bolza, Vorlesungen über Variationsrechnung, Teubner 1909 (Koehler and Amelang 1949).

[Ca] C. Carathéodory, Calculus of Variations and Partial Differential Equations of the First Order, Teubner, Berlin. 2 vols. 1935: English transl., Holden-Day, 1965, 1967. MR 33:597; MR 38:590

[Ce] L. Cesari, Optimization-Theory and Applications, Springer, New York, 1983. MR 85c: 49001

[CV1] F. H. Clarke and R. B. Vinter, Regularity properties of solutions to the basic problem in the calculus of variations, Trans. Amer. Math. Soc. 289 (1985), 73-98. MR 86k:49020

[CV2] _ Existence and regularity in the small in the calculus of variations, J. Differential Equations 59 (1985), 336-354. MR 87a:49014

[Da] A. M. Davie, Singular minimizers in the Calculus of Variations in One Dimensional, Arch. Rational Mech. Anal. 101 (1988), 161-177. MR 89c:49002

[ET] I. Ekeland and R. Temam, Convex Analysis and Variational Problems, North-Holland, Amsterdam, 1976. MR 57:3931b

[FS] W. Fleming and M. Soner, Controlled Markov Processes and Viscosity Solutions, SpringerVerlag, New York, 1993. MR 94e:93004

[L] M. Lavrentiev, Sur quelques problèmes du calcul des variations, Ann. Pura Mat. Appl. 41 (1926), 107-124.

[Ma] M. Mania', Sopra un esempio di Lavrentieff, Boll. Un. Mat. Italiana 13 (1934), 147-153.

[S1] M. A. Sychev, On the question of regularity of the solutions of variational problems, Russian Acad. Sci. Sb. Math. 75 (1993), No 2.

[S2] - On a classical problem of the calculus of variations, Soviet Math. Dokl. 44 (1992), 116-120.

[S3] Lebesgue measure of the universal singular set for the simplest problems in the calculus of variations, Siberian Math. J. 35 (1994).

[T] T. Tonelli, Fondamenti di calcolo delle variazíoni, vol. II, Zanichelli, Bologna, 1921.

Institute of Mathematics of the Russian Academy of Science (Siberian Department), 630090 Novosibirsk, Russia

E-mail address: masychev@math.nsc.ru

Department of Mathematics, Carnegie Mellon University, Pittsburgh, Pennsylvania 15213

E-mail address: vm09@andrew.cmu.edu 